\title{
Lista de Revisores / List of reviewers 2011
}

Abu-Hijleh, Marwan F. (Reino de Bahrain)

Aliendo; Mirta M. (Argentina)

Armand Ugon, Gustavo (Uruguay)

Aughsteen, Adib A. (Jordania)

Ávila, Rodolfo E. (Argentina)

Baca, Vaclav (República Checa)

Ballesteros Acuña, Luis E. (Colombia)

Barut, Cagatay (Turquía)

Campero, Abraham (Argentina)

Caorsi, Carlos (Chile)

Carmichael, Stephen (U.S.A.)

Caussa, Lucas I. (Argentina)

Cheng, A. C. (China)

Chcanovic, Bruno (Brasil)

Cimpean, Anca María (Rumania)

Ciocca, Daniel (Argentina)

Criado del Río, Luis E. (Argentina)

Espósito, Evangelina (Argentina)

Feigl, Georg (Austria)

Ferraris, Sara (Italia)

Fogg, Quentin (Escocia - Reino Unido)

Haviarová, Zora (Eslovaquia)

Ihunwo, Amadi O. (Sudáfrica)

Janevska, Vesna (Macedonia)

Kachlik, David (República Checa)

Kilic, Cenk (Turquía)

Martelotto, Gladys (Argentina)
Martinez Benia, Fernando (Uruguay)

Mendez Oliveri, Gonzalo (Chile)

Merila, Mati (Estonia)

Natsis, Konstantinos (Grecia)

Kayak, Soubhagya R. (India)

Neto Toledo, Joao (Brasil)

Okan, Bilge (Turquía)

Otegui, Gustavo (Argentina)

Ozsoy, Nesrin (Turquía)

Parisi, Sebastián A. (Argentina)

Piagkou, Mara (Grecia)

Plock, Jan (Alemania)

Prados Salazar, José Carlos (España)

Repetto, Emanuel (Argentina)

Rezzani, Rita (Italia)

Seetharam Rao, K. P. (British West Indies)

Spinner, Robert J. (U.S.A.)

Sumathylatha, M. S. (India)

Tekdemir, Ibrahim (Turquía)

Thwin, San S. (Malasia)

Trefilio, Diego E. (Argentina)

Troupis, Theodore G. (Grecia)

Vaida, Monica A. (Rumania)

Vlajkovic, Slobodan (Serbia)

Voss, Ken A. (U.S.A.)

Yücel, Ferruh (Turquía) 